\title{
DISCUSSION OF "PROBABILITY MODELING AND THINKING: WHAT CAN WE LEARN FROM PRACTICE?” BY PFANNKUCH ET AL.
}

\section{BETH CHANCE AND SOMA ROY, CALIFORNIA POLYTECHNIC STATE UNIVERSITY-SAN LUIS OBISPO, USA}

We thank the authors for their painstaking work extracting and analyzing the probabilistic thinking of these practitioners and experts. Although we agree with the limitations of this sample and the need for replication of such investigation in other settings, we did find several of the comments to be very much in accord with our own observations in teaching introductory statistics at the university level and in developing new curricula focused on using simulation-based approaches to learning the reasoning of statistical inference.

As we have switched our introductory statistics course to one centered on using simulation to model the randomness inherent in random sampling and random assignment to build student intuition of $p$-values, we have found one of the main weaknesses of our tertiary students is concrete understanding of modeling. Many of our students enter the course without the ability to differentiate between models and data, simulation vs. reality. Some of this appears to stem from a lack of appreciation of the utility of models, lack of deep understanding of the role of randomness, and the expected difficulties in abstraction. We hope this paper brings to the forefront the very large need to better develop students' intuition of models from very young ages.

This lack of understanding of modeling also impacts students' abilities to understand the role of simulation. This manifests itself in students' struggles with understanding the assumptions underlying the simulation, and how to use these simulated results to answer statistical questions. It can take some students a while to understand the goal of comparing the one set of observed results to what "could have happened" according to a specific simulation model. Too often our students cannot distinguish among simulation strategies, often equating "anything done on the computer" with a "simulation", or believing "repeated sampling" corresponds to repeating the study again. Students need more practice, as advocated for example by the CATALST curriculum (<http://www.tc.umn.edu/ catalst/>, see also Garfield, delMas, and Zieffler, 2012) and now feasible with tools such as TinkerPlots (Konold \& Miller, 2011) with building probability models themselves, in thinking deeply about the assumptions behind those models, and seeing the consequences when the assumptions are incorrect. How often in primary and secondary instruction are students asked to question, critique, and test models (in general and in the context of probability models), or to see the faulty inferences when inappropriate models are applied? At what ages should we be expecting this of our novice learners? What are the essential skills and understanding for students to effectively perform a "sniff test"?

Some additional questions our curriculum work has raised for us include: When and how do we get students to build statistical models at very early stages of learning? In our college-level classes we introduce chance models via coin tossing. Is this model accessible enough to expect even younger students to develop for themselves? It appears we can utilize this model for quite a while without necessitating in-depth study of the binomial distribution, though we still want students to understand the assumptions (e.g.,

Statistics Education Research Journal, 15(2), 38-45, http://iase-web.org/Publications.php?p=SERJ (C) International Association for Statistical Education (IASE/ISI), November, 2016 
independence) behind the model. How do we then best transition to more complicated models? How much of this initial learning should utilize tactile simulations vs. computer micro-worlds vs. black boxes? How critical are the visual components of some simulation tools in helping students develop the ability to "see structure"? Is asking students to write "pseudo-code" explaining the steps of the simulation worthwhile? Sufficient? It would be interesting to learn more from these practitioners how they were able to develop those skills.

A second message that struck us was the need to integrate probabilistic and statistical thinking. Students need to understand the contrast between inductive and deductive reasoning and what each brings to bear, but too often there still seems to be a push to separate probability instruction from statistics instruction, rather than integrating the two together. We believe that integrating probability concepts into statistics curricula ensures that students understand and appreciate how probabilistic thinking informs statistical thinking. Thus, rather than a "probability theory first" paradigm or a "statistics without probability" response, we advocate teaching the probability tools as they are needed to answer interesting statistical questions (e.g., Chance \& Rossman, 2015). We heartily agree with the authors' call for using applications to help develop probabilistic thinking, at all levels, with a focus on making better decisions. Similarly, research in how probabilistic and statistical thinking are developed should also be integrated and mutually informing. Lessons can also be learned from instructional tools being used to help students understand concepts of systems and random processes as in engineering education.

Another area in which we would like to see expansion of such investigations is a focus on the natural intuition of young children and how instruction in chance and models can build on what they bring to the classroom and how improved reasoning can be naturally developed towards the goals advanced in this paper. Although the practitioners' thinking provides an ideal model, we also need to consider what the non-expert needs to know to be able to make reasonable decisions in the presence of uncertainty. In our classroom experiences, we have used "loaded dice" (only sums of 7 and 11 can be rolled, though this is not immediately obvious to the students) to help students realize they often do reason inductively, but we have found it difficult to help them transition to wanting to know what chance "looks like" in other situations. For example, whereas students seem to understand how coin tossing can help them evaluate whether 7 out of 8 heads is an unusual outcome, many students struggle with making the transition from a simple 50-50 chance model to one where "success" and "failure" are not equally likely. How can we help shape such students' intuition on what to model and how such a model is helpful to them? Although we agree that we should model expert thinking when we teach probabilistic thinking, we also want to be careful about what intuition students are bringing to the classroom. We agree that the theoretical mathematical model can reinforce empirical observation, but we hope for more investigation on when transitions to mathematical models (symbols and equations) can or should take place. And can we develop students' ability to reason probabilistically, even if they are not yet ready for the mathematical theoretical complement?

Finally, we very much appreciate the concrete examples provided in this paper of how probability modeling is used by practitioners. We hope additional examples can be made available, especially for instructors seeking to explain and motivate the use of such models. The next question for us as statistics educators is how can we implement what we have learned from these practitioners to guide our students' understanding of probability modeling? How can we best build student intuition at the beginning of learning, rather than waiting for students to base their intuition on years of prior 
experience? Whereas we have found the use of case studies very effective, they can also be time consuming and ultimately limiting in enabling students to generalize. How else can we help students (from varying disciplines) see the role of context in their decision making without getting bogged down in it?

We expect this paper to lead to many fruitful conversations and areas of research and we appreciate the authors' description of a framework on which to build such investigations. Our hope is that the pervasive theme of chance models will soon reach the pinnacle of importance in statistics education along with variation..

\section{REFERENCES}

Chance, B., \& Rossman, A. (2015). Introduction to Statistical Concepts, Applications, and Methods ( $3^{\text {rd }}$ edition). Online: www.rossmanchance.com/iscam3/

Garfield, J., delMas, R., \& Zieffler, A. (2012). Developing statistical modelers and thinkers in an introductory, tertiary-level statistics course. ZDM-The International Journal on Mathematics Education, 44(4), 883-898.

Konold, C., \& Miller, C.D. (2011). TinkerPlots 2.0: Dynamic data exploration. Emeryville, CA: Key Curriculum.

\section{DOUG SHAW, STATISTICIAN, RETIRED, AUSTRALIA}

I appreciate the opportunity to read and comment on this paper. The authors are to be congratulated on the excellent job they have done in synthesising the material obtained through their interviews.

My experience in a working life as an applied statistician has been in consulting and collaboration. My perspective is therefore that of a practitioner, not that of a theoretician or of an educator.

The reality for a practitioner is that, almost invariably, one is applying known models and known methodologies to obtain the required results. Reporting deadlines and project budgets generally preclude the luxury of developing new models or new methodologies. The important skills set that the practitioner in this situation brings to the table are:

1. Familiarity with a wide range of models and methodologies, and an understanding of the problem and context that enables selection of a model/methodology likely to be appropriate for that problem.

2. A thorough understanding of the assumptions underpinning the selected model/methodology. This understanding includes not only knowing the assumptions that support the theory, but also knowing which assumptions are critical, and how to verify that these critical assumptions are met.

As a simple example of criticality of assumptions, we are all familiar with the assumption that "the data are normally and independently distributed." A great deal of research and experience has shown that, in many areas of application, the data can deviate quite markedly from normality without materially affecting the outcome. Deviation from independence is, on the other hand, quite a serious matter.

The interviewees, via the authors, have emphasised the importance of the skills in 1. above, but perhaps have not given sufficient prominence to those in the second point. The accumulation of this knowledge about underpinning assumptions, their criticality, and their verification is vital if one is to become an effective practitioner.

“... accumulation of this knowledge..." turns attention to the educational and developmental aspects of the paper. The interviewees are uniformly adamant about the 
necessity for exposure to many and varied problem contexts, so that the abilities to "see structure" and "apply structure" can be developed. Students should be exposed to many problem contexts, so that can learn to identify common underlying structures and apply models appropriate to those structures. (This dictum applies to statistical modelling with as much force as it does to probability modelling.) But how do we achieve this within a university program? There would seem to be major difficulties.

The first difficulty is simply the time available. Given that the student has other requirements to meet (and there was consensus that theoretical aspects were important), there would be limited time available for the proper presentation of problems in context, for the student to work on the problems, and for the outcome of the work to be discussed and assessed.

A second difficulty concerns the availability of people capable of leading and guiding the students during their exposure to many and varied problem contexts. Just giving the students sufficient detail about the problem context, and doing it in a convincing manner, requires that the lecturers/leaders have themselves had exposure to many and varied contexts. It is unlikely that there will be sufficient lecturers/leaders with that sort of background.

The interviewees generally concurred that something like "intuition" was an essential component of being a successful probability modeller. All of the interviewees appear to be successful probability modellers (in that they identify themselves as probability modellers), so it is difficult to infer for a wider group that this "intuition" was critical for success. If it is critical, how is it to be fostered and developed?

The authors perhaps missed an opportunity to elicit information from the interviewees about their path to their current status as a probability modeller. We have Rose's comments that she did not feel she was a statistician when she completed her $\mathrm{PhD}$, but now, 15 years on, she feels she is one. When did she first feel that she was a statistician, and what made her realise this? If this information was available for all the interviewees, we could obtain some feeling for the dimensions of the task of educating successful probability modellers.

Again, I thank the authors for their careful and detailed work, and the editorial group for the opportunity to comment.

\section{LISBETH KAISERLIAN CORDANI, INSTITUTO DE MATEMÁTICA E ESTATÍSTICA, UNIVERSIDADE DE SAÕ PAULO (IMEUSP), RETIRED, BRAZIL}

It is interesting reading an article with a qualitative approach, a harvest with which I'm not very familiar. But I am aware that many high-level qualitative research articles are being produced, as is the case of this article of Pfannkuch et al. To investigate the mindset of researchers with great expertise and to try to address student learning through these cognitive strategies is a very interesting approach, but there is one crucial point to remember - the necessary training of new university students-for this strategy to be used. I will return to this point later.

We can go to a dictionary to see the entry for 'probability,' which will give information to ordinary citizens, describing various notions-frequentist, classical, Bayesian, axiomatic. We can also think of probability in an informal way, which derives from the way that ordinary people see the world, connected, for example, to the ideas developed in the text of Kahneman, Slovic, and Tversky (1982), which presents heuristics and biases linked to probabilistic reasoning. Often our choices are intuitive and we do not always make decisions with less risk even if we are risk averse. More formal definitions 
are found in texts of probability, with varying degrees of sophistication depending on the audience to whom the book is intended.

Linking the two ways of seeing probability, we can mention Lindley's (1985) statement: "Probability has no existence outside the individual, as the length of a table. Probability expresses the relationship between an individual and the world in which it is contemplated."

Therefore, there is no reason not to expose students to the probabilistic reasoning from the beginning of schooling, as well to the statistics, given the close link between these areas that help the advancement of science in the various fields of knowledge. However in many countries this is not done.

The paper of Pfannkuch et al. quotes Biehler (1994), who discusses the tension between probability and statistics, commenting that this is not new. It is as if there were two different cultures. It is possible to rescue two philosophical currents of the seventeenth century that could be considered as precursors of this tension: the Rationalism of Descartes and the Empiricism of Bacon. For the former, roughly speaking, knowledge-ability that man has within himself-is created from reason. According to Descartes, from basic assumptions about the very genesis of the phenomenon the scientist must first deduce the model and then test it experimentally. Bacon, probably inspired by Aristotle ("Nothing is in the intellect that has not passed through the senses"), adopts the principle that all knowledge is derived from an empirical basis and defends the experimental method, without a priori theory. He states that truth would appear from the simple juxtaposition of data, collected carefully. These two approaches logically alternate in the production of knowledge. Probability and statistics are an intrinsic part of the process, the former involved in the rationalist method and the latter in the empiricist method. In a way, this is linked to the quotation of Doerr and Pratt (2008) that Pfannkuch et al. present.

In the article, respondents who are experts in their domain areas, and who have strong interest in the subject in which they work, alternate between the two approaches described above. They are interested in optimizing their decisions to take advantage either of an economic or an academic activity. The paper contains examples of both abstract research par excellence, for which the world of coins and data has an interest itself, and also more practical or theoretical research, each with its own approach, strategies, and mental models. In summary all kinds of research.

But returning to the case of the student's education: how does this approach facilitate probability and statistics learning? In particular, what type of new university students would find this approach adequate? As we see in the article, the authors are from New Zealand, a developed country, whose national curriculum can be found at http://nzcurriculum.tki.org.nz/The-New-Zealand-Curriculum.

A quick analysis of this curriculum, with eight levels for the 13 years of schooling, reveals consistent reference to Mathematics and Statistics. This already shows an intention to include probability and statistics in elementary school, which is not always found in basic school curricula. At all levels, the goal is to make students think mathematically and statistically. Statistical Investigation, Statistical Literacy, and Probability are presented at all eight levels, producing a knowledge platform for the area that enables the use of progressively more complex components in order to develop a student with a specialized background in this type of reasoning.

By Level 8 (the last years of school) we see applied situations involving Binomial, Poisson, and Normal distributions. Here we understand that the design of Pfannkuch and colleagues' article presupposes a beginning university student with this background, which makes sense of their discussion. Why? Because the student has experienced 
investigative questions, data collection procedures, and simulations that indicated paths to modeling. So the understanding that probability and statistics are important for the advancement of knowledge is presented from the beginning, and familiar structures and more complex models can be developed. This is an ideal situation for the article; many students will engage in this endeavor with dedication, even if there may be some who lack motivation to investigate problems that are not their own or to develop strategies that will not bring them professional reward. Students will have the mental preparation for this, if they want to develop the theme.

Thinking of another reality, for example the Brazilian reality, it would be impossible to address the issue with this level of depth in probability and statistics with regular university students, because they do not have this level of background. An initial analysis of texts that Brazilian students utilize during pre-university education shows the following problems: an approach to probability that is only manipulative, without creativity; statistics and probability with no link between them; probability with no link with decision-making; work with statistics presented in an instrumental way with little or no discussion about variability, just calculation.

Countries such as Brazil are too far from the reality envisaged in this paper, but of course there are others in which these ideas can be used. There is a global movement for curriculum change (even in Brazil), which could facilitate the use of this approach in the medium term.

I congratulate the authors for the careful presentation of various models of reasoning, hoping that it will be a stimulus for the development of the area of probability and statistics in pre-university schooling in other countries. This could give students around the world the possibility of becoming high-level scientists, with knowledge to improve the quality of life on the planet, as well as the opportunity to choose, if they want, the sexiest job of the 21st. century: The data scientist (as Davenport and Patil, 2012, have called it).

\section{REFERENCES}

Biehler, R. (1994). Probabilistic thinking, statistical reasoning, and the search for causes Do we need a probabilistic revolution after we have taught data analysis? In J. Garfield (Chair), Research papers from the fourth international conference on teaching statistics (ICOTS 4), Marrakech, Morocco. Minneapolis, MN: The International Study Group for Research on Learning Probability and Statistics.

Davenport, T.H., \& Patil, D.J. (2012). Data scientist: The sexiest job of the 21st century. Harvard Business Review, 90(10), 70-76.

Doerr, H., \& Pratt, D. (2008). The learning of mathematics and mathematical modeling. In M.K. Heid \& G.W. Blume (Eds.), Research on technology and the teaching and learning of mathematics: A research synthesis (pp. 259-286). Charlotte, NC: Information Age Publishing.

Kahneman, D., Slovic, P., \& Tversky, A. (1982). Judgement under uncertainty: Heuristics and biases. Cambridge University Press.

Lindley, D.V. (1985). Making decisions. Wiley and Sons.

\section{RESPONSE OF AUTHORS TO DISCUSSANTS' COMMENTS}

The authors thank the discussants for their interesting and thoughtful observations on the findings from our paper and the challenging questions they raise about probability education. 
Our goal for this paper was to build a deeper conceptual framework that would be useful for researchers and curriculum developers: a framework encoding desired destinations in preparation for formulating action plans for reaching them. Although specific teaching and learning strategies and curricula will be a vital future step, our hope for this paper was that it might play a similar role to Wild and Pfannkuch's (1999) framework for statistical thinking by stimulating overarching ideas about future pathways for probability education.

We were also thinking in terms of moving beyond the second-class-citizen conception of "probability as the servant of the statistics we teach." In the early years of statistics education, the required probability-model components are too simple to give any glimpse of the power of probability for constructing models of interesting and complex systems. We aim for teaching to facilitate a sense of what probability modeling can do to help us explore how systems work, both for broad audiences and-looking forward-toward developing specializations in probability-modeling to complement statistics specializations. Some parallel development may be desirable, rather than a choice between "probability then statistics" and "probability integrated with statistics."

Complex probability models can be formed by putting together simple building blocks like coins, dice, balls in urns, and spinners in useful ways. All of them provide model-element analogs for a vast array of important applications. There has been emphasis on mathematics about these elements, but they have seen very little use in activities that focus on model construction. The advent of technology such as TinkerPlots (Konold \& Miller, 2011) now makes the power and purpose of models accessible even to middle school students, enabling them to construct models in relatable authentic-type contexts. The potential for building models to help us think about the behavior of complex systems using such simple building blocks is not something many people would ever come up with on their own.

Indeed, Chance and Roy highlight that the concept of chance models needs to gain paramount importance in probability and statistics education on a par with that of variation in statistics education (e.g., Franklin et al., 2007). With the proliferation of data analysis in curricula, chance models are the Cinderella whose time has come to flourish in its own right and also to become integrated with statistics learning. However, as Chance and Roy point out, there are many important research questions to be explored: about how to build students' intuition to see structure, about how to grow students' understanding of the purpose of and concepts underpinning probability modeling, and about what learning experiences and resources students need to have in order to construct models. We compliment Chance and Roy on the breadth of their questions and believe they provide a very good starting point for developing a research agenda in this area.

We agree with Shaw that model assumptions are critical, as we point out in Figure 4, and are an important aspect of learning about probability modeling. Shaw also states that exposing students to many varied contexts may be problematic in terms of time available and teacher expertise. At the tertiary level it may be viewed as problematic, but that should not stop tertiary course programs from rising to the challenge of creating ways of developing students' probability-modeling capacities. Schools can play a role in preparing students to think in probabilistic ways, as Cordani explains in her example of the New Zealand mathematics and statistics curriculum. Indeed work by Lehrer (http://modelingdata.org) in middle schools using chance models and variation in an integrated way to deliver both science and mathematics curricula gives a potential blueprint for how school curricula can change towards including a probability modeling approach. Depending on a country's current curricula and pedagogical practice for statistics and probability, as Cordani points out, a change to include chance and models 
may be too far from reality. A vision and a setting of goals for the future of probability education as proposed in our paper is necessary, however, to stimulate debate and development.

Disruptive technologies such as smartphones and the profusion of "apps" are currently causing profound global and societal changes. We conjecture that all countries may experience or be experiencing disruptive curricula and pedagogical changes within their education systems. We need to be prepared for these changes by having a vision for future possibilities in probability and statistics. With that vision we can work actively through research agendas (e.g., the Statistical Reasoning, Thinking, and Literacy Forums) and statistical associations (e.g., American Statistical Association) in order to have a reform consensus and the conceptual infrastructure in place to instigate and enact changes to curricula.

As we prepare for a future probability modeling approach, which could potentially immerse student learning in simulations and empirical observations, we should not forget that the practitioners we interviewed were very definite that mathematics was a core building block (see Figure 5). Therefore mathematics theory should play an important complementary role in developing chance and models in learning and in curricula.

\section{REFERENCES}

Franklin, C., Kader, G., Mewborn, D., Moreno, J., Peck, R., Perry, M., \& Scheaffer, R. (2007). Guidelines for assessment and instruction in statistics education (GAISE) report: A preK-12 curriculum framework. Alexandria, VA: American Statistical Association. Retrieved from http://www.amstat.org/education/gaise/

Konold, C., \& Miller, C.D. (2011). TinkerPlots 2.0: Dynamic data exploration. Emeryville, CA: Key Curriculum.

Wild, C.J., \& Pfannkuch, M. (1999). Statistical thinking in empirical enquiry (with discussion). International Statistical Review, 67(3), 223-265. 